\title{
Contribution to the numerical study of the thermal gradient during the fabrication of composite materials by the RTM process.
}

\author{
Soukayna ELYOUSSFI ${ }^{1}$, Abdellatif EL OUJDI ${ }^{1}$, Aouatif SAAD ${ }^{1}$, Mohamed HATTABI ${ }^{2}$ \\ \& Adil ECHCHELH ${ }^{1}$. \\ \{ Email adress 1: ssoukaynaelyou@gmail.com, Email adress 2: abdellatif.eloujdi@gmail.com, Email \\ adress 3: saad_aouatif@yahoo.fr, Email adress 4: mohamdhattabi@hotmail.com, Email \\ adress5: adilechel@gmail.com\} \\ ${ }^{1}$ Laboratory of Materials and Energy, faculty of sciences, University of kenitra, Morocco. \\ ${ }^{2}$ Department of Mechanical Engineering, ENSEM, University of Casablanca, Morocco.
}

\begin{abstract}
Resin Transfer Molding (RTM) has become one of the most efficient processes in the field of composite materials, among the steps in composite part processing is the curing reaction. In the majority of cases this reaction is of exothermic nature and accompanied by a rise in temperature in the center of the laminate, this leads to the appearance of a thermal gradient. This research work aims to highlight the thermal gradient generated as a result of a cross linking reaction. The objective is to reduce the temperature excess in the Polyester / Glass laminate. By means of a one-dimensional numerical study using the finite- difference method we have shown that the energy balance depends not only on the temperature and the degree of curing but also on several other factors namely: the volume fraction of the fibers and the temperature cycle. The comparison of the results with literature showed a good agreement.
\end{abstract}

Keywords. Resin transfer molding (RTM), thermal gradient, Polyester/Glass, one-dimensional, finite difference, temperature, degree of curing, volume fraction of the fibers.

\section{Introduction}

Since their advent in the thirties and until today composite materials have strongly contributed to the development of our techniques. Among the industrial processes used in the production phase is resin transfer molding RTM. The latter is considered one of the most promising techniques available today; it is able to make a great deal complex shapes with a great mechanical performance, tolerance, dimensional, narrow and high surface finish [1]. The manufacturing of a part by the RTM process can be freely divided into four main stages [2]: fiber preform manufacturing, mold filling, curing and remolding the part.

After the filling of the whole mold, a curing reaction is initiated and is intended to bind the liquid resin to the solid reinforcement. In most cases, this reaction is initiated by a heating of the mold. The exothermic curing reaction is accompanied by an increase in the external temperature. This released heat can thus, if not properly distributed, further promote the curing reaction which once again generates heat; therefore, this gives rise to a temperature gradient in the composite which increases as a result of the low thermal conductivity of the polymer matrices. This thermal gradient in turn causes residual stresses, defects in the final part.

A numerical study of the curing process remains a good way to predict the degree of cure and the temperature distribution. Several attempts have been made to reduce this thermal gradient by optimizing the energy cycle. Nonexhaustive, the work of Choi and al. [2] who manipulated with a temperature profile that represents a constant temperature interval, we observed as a consequence the disappearance of this gradient which is argued by the weak transfer heat in this time interval, so the temperature will not exceed its value at the wall of the mold. Antonucci and al. [3] presents a methodology based on the scale analysis of the energy balance equation to manage the heat generated by the curing reaction. Their approach allows to minimize temperature gradients before the resin solidifies. Loos and al. [4] involved the development of a one-dimensional simulation of a plate using an implicit method of finite difference.

In this study, we have shown that the energy balance depends not only on the temperature and the degree of cure, but also on several other intervening factors characterizing both the matrix and the reinforcement, namely: the fiber 
volume fraction and the the temperature cycle of the mold wall. In our numerical approach, we have opted for a onedimensional finite difference method to study the temperature transfer in composite and the rate of the curing reaction in the RTM process. To validate our results, we have compared the numerical solutions obtained with the data available in the literature, this gives good agreement.

\section{Mathematical model}

\subsection{Heat transfer equation}

The one-dimensional equation that governs heat transfer in the composite laminate is given as follows [4, 5 and 6$]$ :

$$
\rho C_{p} \frac{\partial T}{\partial t}=k \frac{\partial^{2} T}{\partial z^{2}}+\rho_{r} \cdot\left(1-v_{f}\right) \cdot \Delta H_{r} \cdot \frac{d \alpha}{d t}
$$

$k, \rho, v_{f}, \Delta H_{r}, \alpha$ and $C_{p}$ are respectively the thermal conductivity, the density, the volume fraction, the heat reaction, the degree of cure of resin and the heat capacity. According to Choi et al. [2], the thermal properties of the resin, the fiber and the cured part are defined as follows:

The mass fractions of the resin $\left(w_{r}\right)$, the cured product $\left(w_{p}\right)$ and the fibers $\left(w_{f}\right)$ are respectively given by $[2,7,8]$ :

$$
\begin{gathered}
w_{r}=w_{r 0} \cdot(1-\alpha) \\
w_{p}=w_{r 0} \cdot \alpha \\
w_{f}=w_{f 0}
\end{gathered}
$$

The volume fraction of the resin $\left(v_{r}\right)$, the cured product $\left(v_{p}\right)$ and the fibers $\left(v_{f}\right)$ are given respectively by $[2,7]$ :

$$
\begin{gathered}
v_{r}=w_{r} \frac{\rho}{\rho_{r}} \\
v_{p}=w_{p} \frac{\rho}{\rho_{p}} \\
v_{f}=\frac{\rho}{\rho_{f}}
\end{gathered}
$$

The expressions of density, specific heat capacity and thermal conductivity are given, respectively, by [7, 9]:

$$
\begin{gathered}
\frac{1}{\rho}=\frac{w_{r}}{\rho_{r}}+\frac{w_{p}}{\rho_{r}}+\frac{w_{f}}{\rho_{f}} \\
C_{p}=w_{r} \cdot C_{p, r}+w_{p} \cdot C_{p, p}+w_{f} \cdot C_{p, f} \\
\ln k=V_{r p} \cdot \ln k_{r p}+V_{f} \cdot \ln k_{f}
\end{gathered}
$$

\subsection{Kinetic model}

The equation of the curing rate reactions used in our case is defined as follows $[10,11]$ :

$$
\frac{d \alpha}{d t}=\left(K_{1}+K_{2} \alpha^{m}\right)(1-\alpha)^{n}
$$

With:

$K_{i}=A_{i} \cdot \exp \left(-\frac{E_{i}}{R T}\right)$

Or: $\mathrm{i}=1,2$

From where $\mathrm{T}$ is the temperature, $\alpha$ is the degree of cure and $\frac{d \alpha}{d t}$ indicates the reaction rate, $K_{i}$ are reaction rate constants. $E_{i}$ present the activation energy, $\mathrm{R}$ the universal gas constant, $\mathrm{m}$ and $\mathrm{n}$ are empirical exponents in the cure kinetic model.. 


\section{Boundary conditions}

Initial condition ( $\mathrm{t}=0): T=T_{0}$

The side wall: $\quad T=T_{w}(t)$

In the center of the wall: $\quad \frac{\partial T}{\partial n}=0$

For the polymerization of Polyester / Glass composite laminate, the initial condition [11]: $\alpha=\alpha_{0}$ at $\mathrm{t}=t_{0}$.

\section{Numerical model}

In our study we have opted with the explicit finite difference method to discretize the two equations (1) and (7). Temperature and degree of cure distributions in the entire domain are calculated at each time step increment as a function of the temperature history of the curing cycle [12].

The discretization of the curing equation by the finite difference method gives:

$$
\alpha_{j}^{t+\Delta t}=\left({\frac{d \alpha^{t+\Delta t}}{d t_{j}}}^{t}+\alpha_{j}^{t}\right) . \Delta t
$$

The stability condition used in this work is expressed as follows [13]:

$$
\Delta t \leq \Delta z^{2} /(2 . a)
$$

The discretization of the heat equation by the finite difference method is given as follows:

$$
\frac{T_{j}^{i+1}-T_{j}^{i}}{\Delta t}=\frac{k}{\Delta z^{2}}\left(T_{j+1}^{i}-2 T_{j}^{i}+T_{j-1}^{i}\right)+\rho_{r} .\left(1-v_{f}\right) \cdot \Delta H_{r}\left(\frac{d \alpha}{d t}\right)_{j}^{t+\Delta t}
$$

This gives:

$$
T_{j}^{i+1}=T_{j}^{i}+\mathrm{a} \cdot \Delta t / \Delta z^{2}\left(T_{j-1}^{i}-2 T_{j}^{i}+T_{j+1}^{i}\right)+\rho r \cdot\left(1-v_{f}\right) \cdot \Delta H_{r} \cdot \Delta t / \Delta z^{2} \cdot\left(\frac{d \alpha}{d t}\right)_{j}^{t}
$$

\section{Results and Discussions}

\subsection{Validation of results}

Considering a flat rectangular mold with the dimensions of $10 \mathrm{~cm}$ in length and $1 \mathrm{~cm}$ in thickness. The mold is filled with fiber glass preforms and saturated with the polyester resin which is heated through the mold wall to initiate curing. For the polyester / glass composite laminate, we adopted in our work the kinetic parameters adopted by Choi et al. [2] illustrated in Table 1, and the thermo-physical properties shown in Table 2.

Table 1. Cure kinetics for Glass / polyester [2].

\begin{tabular}{lr}
\hline $\boldsymbol{k}_{\mathbf{1}}(\mathbf{1} / \boldsymbol{s})$ & $5,68667 . \mathbf{1 0}^{\mathbf{1 2}}$ \\
$\boldsymbol{k}_{\mathbf{2}}(\mathbf{1} / \boldsymbol{s})$ & $8,61167 . \mathbf{1 0}^{\mathbf{8}}$ \\
$\boldsymbol{E}_{\mathbf{1}}(\mathbf{c a l} / \mathbf{M})$ & 25570 \\
$\boldsymbol{E}_{\mathbf{2}}(\mathbf{c a l} / \mathbf{M})$ & 17930 \\
$\mathbf{n}$ & 1,42 \\
$\mathbf{m}$ & 0,58 \\
$\Delta \mathbf{H}(\mathbf{c a l} / \mathbf{M})$ & 47,8 \\
\hline
\end{tabular}


Table 2. Material properties of Glass / polyester [2,7].

\begin{tabular}{|l|c|c|c|}
\hline Materials & $\boldsymbol{\rho}\left(\mathbf{g} / \mathbf{c m}^{\mathbf{3}}\right)$ & $\boldsymbol{C}_{\boldsymbol{p}}(\mathbf{c a l} / \mathbf{g . K})$ & $\mathbf{k}(\mathbf{c a l} / \mathbf{c m} . \mathbf{s . K})$ \\
\hline $\begin{array}{l}\text { Uncured } \\
\text { Polyester }\end{array}$ & 1.10 & 0.45 & $4.05 .10^{-4}$ \\
\hline Cured Polyester & 1.20 & 0.45 & $1.54 .10^{-4}+9.46 .10^{-7} T$ \\
\hline Class fiber & 2.54 & 0.199 & $2.07 .10^{-3}$ \\
\hline
\end{tabular}

The numerical model that we developed with MATLAB calculation code for the polyester resin and fiberglass system is based on the coupled resolution of the heat equation and the rate of the curing reaction. This allows describing the evolution of the temperature and the chemical kinetics of the resin. This coupling makes possible to calculate both the temperature and the degree of curing $\alpha(T, \alpha)$ at each point in the thickness of the laminate in the mold and at each moment [14].

From the results illustrated in figure 1 and 2 we can observe that the temperature and the degree of cure develops very quickly at the center of the polyester / glass composite laminate which causes an exothermic peak. We then validated our results with Choi et al. [2] and a good agreement is observed.

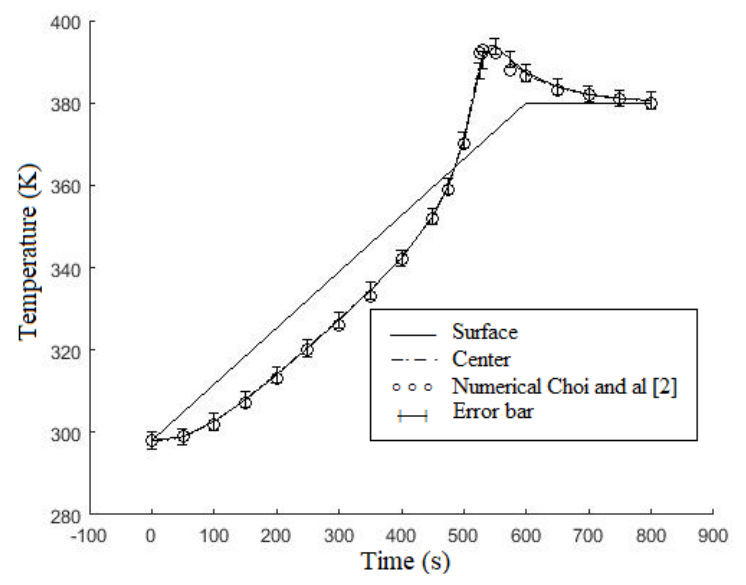

Fig.1. Temperature profile at the central node for the glass / polyester laminate.

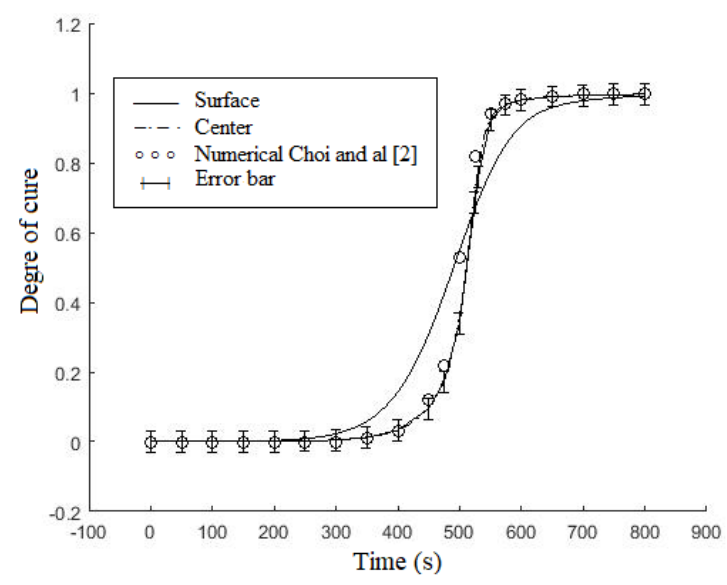

Fig. 2. Degree of cure at the central node for glass / polyester laminate. 
Thermal gradients during the RTM process give rise to several concerns, among which: residual stresses that could lead to shrinkage and lack of dimensional tolerance, thermal stress that can deform the composite part....

The following part presents the one-dimensional numerical model used to simulate the curing stage and to predict the temperature profile in the RTM process and in particular for the minimization of the indesirable thermal gradient .

\subsection{Strategies to reduce the thermal gradient in the composite laminate}

\section{$>$ Optimization of the temperature cycle}

In order to reduce and control thermal gradients in the RTM process for a polyester resin and fiber glass, we have opted for the optimization of the temperature cycle. The variables that have to be controlled are the degree of curing or the adjustment of the temperature boundary conditions of the mold wall. In our case, the methodology used is based on adjusting the temperature boundary conditions of the mold wall; this was done in two steps: In the first step, which is called the conduction zone, the mold wall temperature was raised to a plateau and held until the temperature across the thickness is close to uniformity. In the second step, called the reaction zone, the temperature of the mold wall was raised to a higher temperature to complete the cure reaction. For a clearer view, figures 3 and 4 show the temperature evolution in time for the surface and center of the composite laminate.

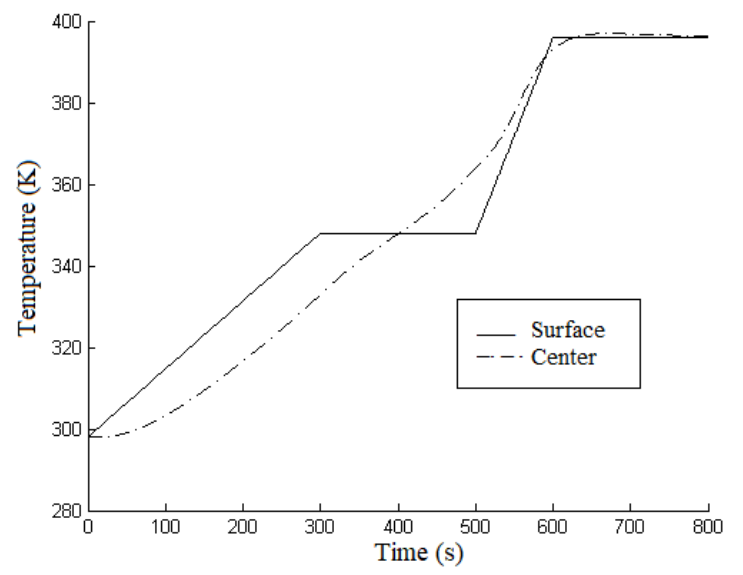

Fig. 3. Temperature profile at the central node for the glass / polyester laminate in the case of an optimized cure cycle.

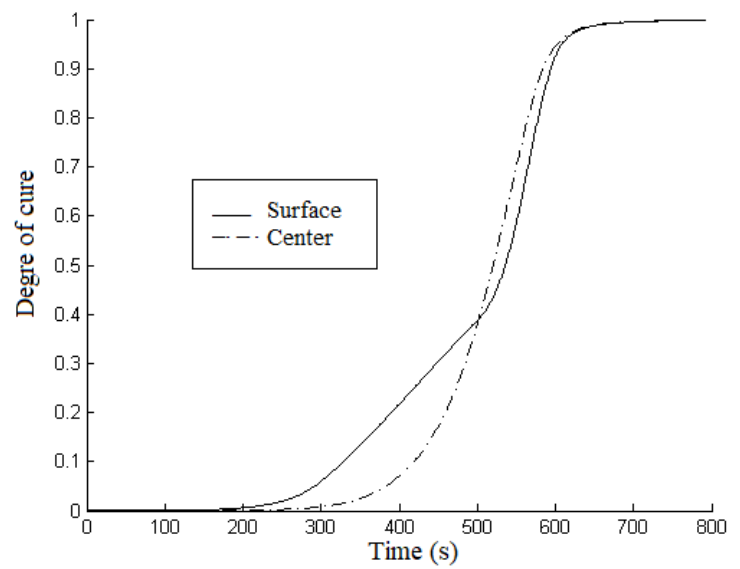

Fig. 4. Degree of cure at the central node for the glass / polyester laminate in the case of an optimized cure cycle.

The results of this procedure as showed in Figures 3 and 4 are very satisfactory with a disappearance of the thermal gradient. Thus the temperature will not exceed its value at the wall of the mold. In return, the curing process takes a constant time to complete. On the other hand, the curing reaction develops uniformly giving rise to a minimum of residual stresses in the final piece.

\section{Choice of the fiber volume fraction}


One of the most important parameters affecting the properties of composites is the fiber volume fraction. In our work we have studied the effect of varying the fiber properties especially fiber content on the temperature and curing rate profile.

Figures 5 and 6 show the profile of the temperature and the degree of curing in the center of the mold for differents volume fraction of fibers: $v_{f}=0.2, v_{f}=0.4$ and $v_{f}=0.5$.

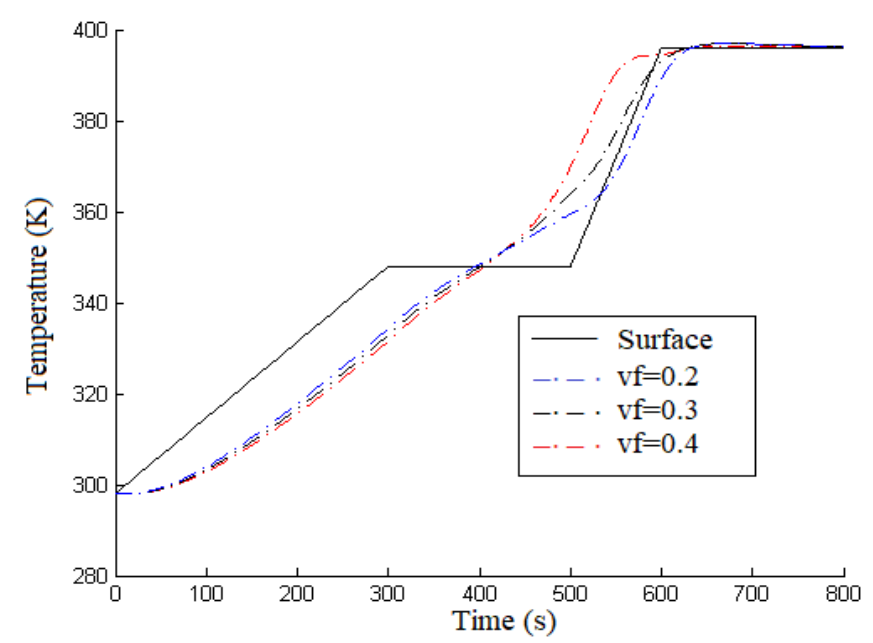

Fig.5. Profile of the Temperature at the central node of the Polyester /Glass composite laminate in the case of optimized polymerization cycle for different cases of volume fraction of fibers.

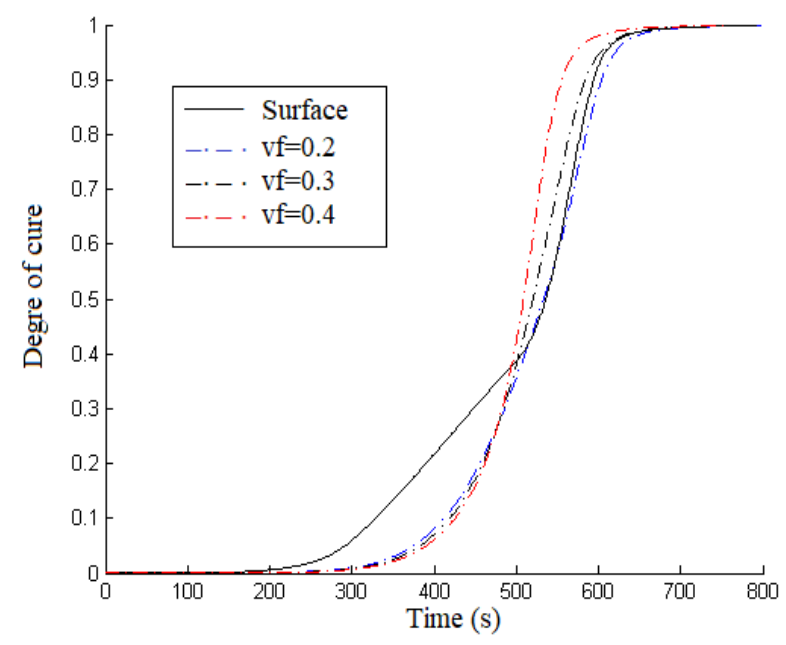

Fig.6. Degree of curing at the central node of the Polyester /Glass plate in the case of an optimized polymerization cycle in different cases of fiber volume fraction.

It's clear from the figures 5 and 6 that by increasing the amount of fiber present in the system, the peak of exothermic reaction was reduced and the thermal conduction was improved. This was explained by the fact that the fiber glass had a higher thermal conductivity than the polyester resin. It is thus of great interest to inrease the fiber volume fraction of the reinforcement used to first minimize the thermal gradient and then to increase the mechanical performance of the composite part. 


\section{Conclusion}

In this work, we have developed a calculation program under MATLAB, we opted for the explicit finite difference methods in the one-dimensional case applied to the case of a polyester / glass composite laminate at the macroscopic scale. This code has the ability to predict the temperature profile and degree of cure in the mold. Thanks to our numerical simulations, we have found that the thermal gradient, which is detrimental to the quality of the final product, can be considerably reduced through a series of preventive measures, among others: The optimization of the temperature cycle in the mold and the choice of the convenient volume fraction of the fibers.

With the results we obtained by our calculation code, the methodology developed proved to be able to effectively solve the problem of thermal gradients. We are considering in the following, the research of other methods that can reduce the thermal gradient in order to improve the mechanical properties of the composite part.

\section{References}

[1] A. Saad, A. Echchelh, M. hattabi, M. El Ganaoui, 20111, An Improved Computational Method For Non Isothermal Resin Transfer Moulding Simulation, Thermal sciences, 15, pp. 275-289.

[2] Choi, M.A., Lee, M.H., Lee, S.J.: Numerical studies during cure in the resin transfer molding. Vol 6, pp. 211218. Korea polymer journal, (1998).

[3] Vincenza, A., Michele, G., Kuang-Ting, H., Suresh, G.A.: A methodology to reduce thermal gradients due to the exothermic reactions in composites processing. Vol 45, pp.1675-1684. International Journal of Heat and Mass Transfer, (2002).

[4] Loos, A.C., Springer,G.S.: Curing of epoxy matrix composites. Vol 17, pp. 135-169. Journal of Composite Materials, (1983).

[5] Yi, S., Hilton, H.H., Ahmad, M.F.: A finite element approach for cure simulation of thermosetting matrix composites. Vol. 64, pp. 383-388. Civil-Comp and Elsevier Science, (1997).

[6] Kim, J.S., Lee, D.G.: Development of an Autoclave Cure Cycle with Cooling and Reheating Steps for Thick Thermoset Composite Laminates. Vol. 31, pp. 2264-2282. Journal of Composite Materials, (1997).

[7] Han, C., Lee, D., Chin, H.: Development of a Mathematical Model for the Pultrusion Process. Vol. 26, pp. 393404. Polymer Engineering and Science, (1986).

[8] Aouatif Saad, Adil Echchelh, Mohamed Hattabi and Mohammed El Ganaoui : The identification of effective thermal conductivity for fibrous reinforcement composite by inverse method. Vol. 33(23) 2183-2191. Journal of Reinforced Plastics and Composites, 2014.

[9] Saad, A., Echchelh, A., Hattabi, M. and El Ganaoui, M.: Optimization of the cycle time in resin transfer molding process by numerical Simulation. Vol.31, pp. 1388-1399. , Journal of Reinforced Plastics and Composites, (2012). [10] KAMAL, M.R., SOUROUR, M.R.: Kinetic modelling of epoxy-amine cure process. Vol. 13, pp. 59-64. Polymer Engineering and Science, (1973).

[11] Cheung, A., Yu, Y., Pochiraju, K.: Three-dimensional finite element simulation of curing of polymer composites. Vol. 40, pp. 895-912. Finite Elements in Analysis and Design, (2004).

[12] Bogetti, T.A., Gillespie, J.W.: Two-dimensional cure simulation of thick thermosetting composites. Vol. 25, pp. 239-273. Journal of Composite Materials, (1991).

[13] Goncalvès, E. : Résolution Numérique, Discrétisation des EDP et EDO. Institut National Polytechnique de Grenoble, (2005).

[14] Bogetti, T.A., Gillespie, J.W.: Process-Induced Stress and Deformation in Thick-Section Thermoset Composite Laminates. Vol. 26, pp. 627-660. Journal of Composite Materials, (1992). 